Copyright 2009 IEEE. Published in the IEEE 2009 International Conference on Image Processing (ICIP 2009), scheduled for November 7-11, 2009 in Cairo, Egypt. Personal use of this material is permitted. However, permission to reprint/republish this material for advertising or promotional purposes or for creating new collective works for resale or redistribution to servers or lists, or to reuse any copyrighted component of this work in other works, must be obtained from the IEEE. Contact: Manager, Copyrights and Permissions / IEEE Service Center / 445 Hoes Lane / P.O. Box 1331 / Piscataway, NJ 08855-1331, USA. Telephone: + Intl. 908-562-3966. 


\title{
2-D SHAPE REPRESENTATION USING IMPROVED FOURIER DESCRIPTORS
}

\author{
Jonas De Vylder, Wilfried Philips \\ Ghent University - IBBT - Dept. of Telecommunications and Information Processing \\ St.-Pietersnieuwstraat 41, B-9000 Ghent, Belgium \\ Jonas.deVylder@telin.ugent.be
}

\begin{abstract}
Fourier descriptors (FD's) are widely used shape descriptors. By first warping the scanning speed of the contour before calculating the FD's, the shape approximation can be improved. This approach has never been properly tested on real applications. In this paper we compare these new shape descriptors to the regular FD's. A database of over 400 leaf shapes is approximated using these shape descriptors and compared to the approximation based on FD's. The error of the approximation was measured with the Hausdorff distance and modified Hausdorff distance, resulting for both criteria in an average improvement of more than $10 \%$ over approximation based on FD's.
\end{abstract}

Index Terms - Image shape analysis, Pattern recognition, Shape Representation, Contour based shape descriptors, (Improved) Fourier descriptors

\section{INTRODUCTION}

Compact shape representation is important for segment coding, coding of medical signals, silhouette coding, etc. Compact shape representation is not only interesting for the purpose of data compression, but also for pattern recognition, where it helps to overcome the curse of dimensionality. The required accuracy of representation is lower for these applications, but the power to discriminate between different shapes is more important. Typical applications are classification [1, 2], model based segmentation [3, 4], retrieving similar shapes from databases $[5,6]$, motion analysis, posture analysis, etc. Due to the many applications, shape representation has been a very active field of research, and has lead to a variety in description methods.

In literature, two broad classes of techniques can be distinguished: the region based methods and the contour based methods. In the region based methods, the object is represented by a binary image which pixels represent the object. Parameters, such as statistical moments are computed on this binary image. Methods belonging to this group are quad trees, central moments, Zernike moments, etc. In contour based

This research has been made possible by the Institute for the Promotion of Innovation by Science and Technology in Flanders (IWT). methods, only the points belonging to the contour are used to represent the object. For several of these methods, the contour is parameterized. A wide variety of parameterizations have been investigated in literature, e.g. $(x(t), y(t))$ where $x(t)$ and $y(t)$ are the coordinates of point " $t$ " on the contour, the complex representation of the coordinates $(x(t)+j y(t))$, the angle between three subsequent contour points, the centroid distance $\left(\sqrt{\left(x(t)-x_{0}\right)^{2}+\left(y(t)-y_{0}\right)^{2}}\right.$, where $x_{0}$ and $y_{0}$ are the coordinates of the center of the object), etc. The contour parametrization $s(t)$ can be further processed for shape description. Methods belonging to this group are Chain codes, Fourier descriptors, Curvature Scale Space, etc. For a more thoroughly review on shape description we refer to [7].

In this paper we will further work with discrete contour based methods, i.e. only an finite number $\mathrm{N}$ of contour points $s\left(t_{n}\right), n=0, \ldots, N-1$ are known. This paper is arranged as follows. In Section 2, we will define three metrics which can be used to compare two shapes. In Section 3 the Fourier descriptors are explained. A generalization of the Fourier descriptors are also explained in this section. These new shape descriptors, Improved Fourier descriptors, are tested for the purpose of shape approximation in section 4 . Section 5 recapitulates and concludes.

\section{SHAPE DIFFERENCE METRICS}

In order to compare different representation techniques, a metric to express similarity or dissimilarity of a shape and its approximation is needed. The mean squared distance is a metric which is used to compare contour parameterizations. Consider $s_{1}$ and $s_{2}$ to be the same parameterization of two shapes, the mean squared distance between $s_{1}$ and $s_{2}$ is defined as follows:

$$
d_{m s d}\left(s_{1}, s_{2}\right) \triangleq \frac{1}{N} \sum_{k=0}^{N-1}\left(s_{1}\left(t_{k}\right)-s_{2}\left(t_{k}\right)\right)^{2}
$$

This might be a good measurement for differences between functions, but it is not necessarily a good measurement for difference between shapes. Let $s_{1}$ and $s_{2}$ describe the same shape, but with an other starting point, e.g. $s_{2}\left(t_{k}\right)=$ $s_{1}(k+1)$ with $t_{N}=t_{0}$. Even though the shapes are equal, 
the mean squared distance differs from zero. To overcome this problem, two other metrics are defined in [7]: the Hausdorff distance and the modified Hausdorff distance. For two sets of points $A=a_{1}, a_{2}, \ldots, a_{p}$ and $B=b_{1}, b_{2}, \ldots, b_{q}$ the Hausdorff distance (HD) is defined as follows

$$
d_{H}(A, B) \triangleq \max (h(A, B), h(B, A))
$$

where

$$
h(A, B) \triangleq \max _{a \in A} \min _{b \in B} d(a, b)
$$

With $d$ a given metric function. For our purpose the euclidean distance is used.

The Hausdorff distance is a measurement of the maximum distance between two contours. Whereas the modified Hausdorff distance (MHD) is a measurement of the average distance between two contours. This distance function is given by eq. 2 where

$$
h(A, B) \triangleq \frac{1}{p} \sum_{a \in A} \min _{b \in B} d(a, b)
$$

Here again the euclidean distance is used for $d$.

\section{CONTOUR BASED SHAPE DESCRIPTION}

In this section the concept of Fourier descriptors and there generalization, i.e., the improved Fourier descriptors are explained.

\subsection{Fourier descriptors}

A class of contour descriptors which are widely used, are Fourier descriptors (FD). For the rest of this paper we will assume the contour is parameterized using the complex coordinates. Consider the trigonometric polynomial of order N:

$$
s_{N}(t) \triangleq \sum_{n=-N}^{N} B_{n} e^{j n t}
$$

where $B_{n}$ minimizes the mean squared distance between $s_{N}(t)$ and $s(t)$. The coefficients $B_{n}$ are called the Fourier descriptors. $B_{n}$ is equal to the nth frequency component of $s(t)$ and can easily be computed using the discrete Fourier transform. One of the main advantages of FD's is the fact that if the shape undergoes certain transformations, the FD's change in a simple manner, i.e.

- A translation only affects the FD which corresponds to the zero frequency component, i.e. $B_{0}$, so except of $B_{0}$ the FD's are invariant to translation.

- Scaling the object result in a multiplication of all FD's with a real constant.
- Rotating the object with angle $\theta$ results also in a multiplication, but with $e^{j \theta}$.

- Changing the starting point of $s(t)$ results in a phase shift, so $\left|B_{n}\right|$ are invariant to rotation and change of starting point.

These properties have made FD's popular in a wide range of applications [1, 2, 3, 4, 8, 6, 9, 10]. Note that this FD's are defined for other parameterizations then the complex coordinates as well. A more thoroughly overview of FD's using different parameterizations can be found in [5].

\subsection{Improved Fourier descriptors}

Consider $C(s(t))$, to be the contour described by $s(t)$, and $C(s(\theta(t)))$, where $\theta(t)$ is a warping function, i.e., any strictly monotonous function that maps the interval $[0,2 \pi]$ onto itself. $C(s(t))$ and $C(s(\theta(t)))$ describe the same contour. This opens the possibility of optimizing contour representation by selecting an optimal $\theta(t)$ according to some criterion. The FD's calculate the trigonometric polynomial with the least mean squared distance with $s(t)$. It would be better to calculate the FD's for all warped contours $s(\theta(t))$ and chose the FD's with the smallest error. This results in the following trigonometric polynomial:

$$
s_{N}^{\prime}(t) \triangleq \sum_{n=-N}^{N} G_{n} e^{j n t}
$$

where the coefficients $G_{n}$ minimize the mean squared distance between $s_{N}^{\prime}(t)$ and $s(\theta(t))$ with $\theta$ the most optimal warping function. The $G_{n}$ coefficients are called improved Fourier descriptors (IFD's). Since the IFD's are FD's of $s(\theta(t))$, they have the same properties as FD's concerning translation, scaling, rotation and changes of starting point. Optimizing $d_{M S D}\left(s(\theta(t)), s_{N}^{\prime}(t)\right)$ is equivalent to optimizing $e_{N}=d_{M S D}\left(s(t), s_{N}^{\prime}(\gamma(t))\right)$. This approach has the advantage that it does not need to interpolate between two contour points $s\left(t_{k}\right)$ and $s\left(t_{k+1}\right)$. This results in $s_{N}^{*}(t) \triangleq s_{N}^{\prime} \circ \gamma^{*}(t)$, i.e.

$$
s_{N}^{*}(t)=\sum_{n=-N}^{N} G_{n} e^{j n \gamma^{*}(t)}
$$

with $\gamma^{*}(t)$ the optimal warping function. To solve this optimization problem, an iterative algorithm is proposed in [11]. Consider the discrete case, where $\gamma$ is represented by a multidimensional vector. Then in the nth iteration, this vector is updated by

$$
\gamma_{k}^{n}=\gamma_{k}^{n-1}+\alpha_{n} d_{k}^{n}
$$

Here $d_{k}$ is the difference between the average error and the error at point $k$. This results in optimizing $\gamma$ by spreading the error. The parameter $\alpha_{n}$ is the step size that minimizes $e_{N}$. 
As an initial value for $\gamma$ we choose $\gamma(t)=t$. There is no guarantee the algorithm converges in a finite number of iterations. In practice however, $e_{N}$ generally no longer changes significantly after three iterations. This iterative process requires multiple calculations of $s_{N}(\gamma(t))$ and the error. This can be simplified and speeded up using a decomposition in orthonormal polynomials as suggested in [11].

\section{RESULTS AND DISCUSSION}

\subsection{Data}

To test the ability of IFD's to represent shapes, a database with pictures of leaves was used. This database contains pictures of isolated leafs from six different plant species (circinatum, garryana, glabrum, kelloggii, macrophyllum and negundo). These are color pictures of $512 \times 512$ pixels. The leafs were extracted out of the picture using a threshold on the RGB values. In Fig. 1 an example of the result of this color filter can be seen for each different plant species. In total 436 of these images were used to test the use of IFD's for shape approximation. Each shape was approximated using 10, 20, 30, 40 and 50 IFD's. For comparison all images were also reconstructed using the same amount of FD's.

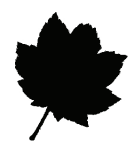

(a) circinatum

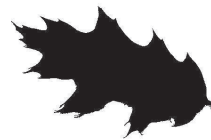

(d) kelloggii

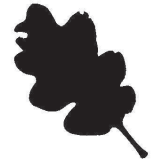

(b) garryana

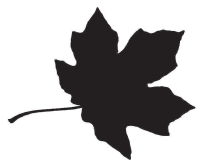

(e) macrophyllum

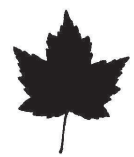

(c) glabrum

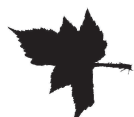

(f) negundo
Fig. 1. Examples of the leaf database after preprocessing.

\subsection{Measurements}

As an example, two shapes were approximated using both improved and regular Fourier descriptors descriptors. In Fig. 2, the results are shown. The first row shows the leafs we want to approximate. In the second and third row there approximation using respectively 10 FD's and IFD's can be seen. In these examples, it is clearly visible that the IFD's approximate the original shape better than the FD's. For both examples, the IFD approximation shows more clearly the leaf incisions. In the first example also the long extension of the leaf stalk is clearly captured by the IFD approximation.

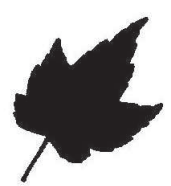

(a) the original shape

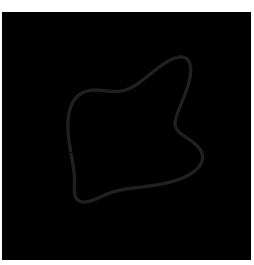

(c) $10 \mathrm{FD}$

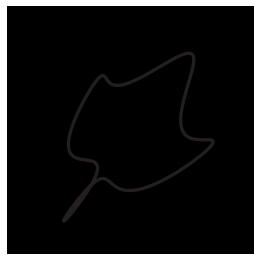

(e) 10 IFD

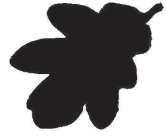

(b) the original shape

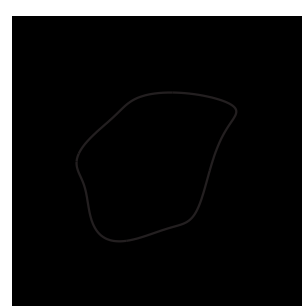

(d) $10 \mathrm{FD}$

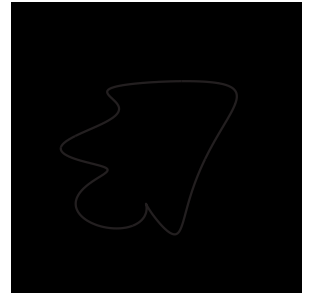

(f) 10 IFD
Fig. 2. Examples of the representation using FD's and IFD's. In the first row the shape we want to approximate is shown. In the second and third row the approximations are shown.

In the top part of Table 1 the average Hausdorff distance can be seen between the original shape and the approximation using FD's. The performance of the FD's depends on the kind of shapes they have to represent, e.g., the class of kelloggii leafs are represented much worse than the class of garryana leafs. It is also clear that the more descriptors are used, the better the approximation. In the bottom part of Table 1 the same measurements are shown, but here IFD's were used instead of FD's. The same remarks as for FD's can be made. The average improvement of the Hausdorff distance of the IFD approximation over the FD approximation is over $14 \%$. Even though some classes are approximated better than others, on average, every class is better approximated using IFD's. Measuring the difference using the modified Hausdorff distance confirms the conclusions that were made using the Hausdorff distance as an error measurement.

In Fig. 3, a histogram is shown which visualizes how many percentage the modified Hausdorff distance improves using the IFD's to approximate the shape instead of the FD's. This histogram incorporates all measurements, i.e., for 10, 20, ..., 50 IFD's. Tests showed that there was no clear difference between histograms which only incorporated approximations using the same amount of descriptors. As we can see in this 
Table 1. The Hausdorff distance between the original and the reconstructed shape using a different number of FD's or IFD's.

\begin{tabular}{l|rrrrrr|r} 
plant & circinatum & garyana & glabrum & kelloggii & macrophyllum & negundo & average \\
\# images & 64 & 84 & 75 & 94 & 82 & 37 & 436 \\
\hline 10 FD's & 37,2 & 32,9 & 34,4 & 47,1 & 42,4 & 28,4 & 38,3 \\
20 FD's & 18,5 & 14,1 & 15,5 & 24,9 & 19,3 & 14,6 & 18,3 \\
30 FD's & 10,0 & 8,9 & 9,9 & 16,4 & 12,1 & 9,6 & 11,5 \\
40 FD's & 7,7 & 6,4 & 7,3 & 12,5 & 8,9 & 7,4 & 8,6 \\
50 FD's & 6,5 & 5,0 & 6,2 & 10,2 & 7,0 & 6,2 & 7,0 \\
\hline 10 IFD's & 31,0 & 30,8 & 27,0 & 46,0 & 41,0 & 27,7 & 35,1 \\
20 IFD's & 16,8 & 13,0 & 14,2 & 23,3 & 18,2 & 13,0 & 16,9 \\
30 IFD's & 9,5 & 6,9 & 9,0 & 15,0 & 10,9 & 7,8 & 10,2 \\
40 IFD's & 7,0 & 4,4 & 6,7 & 10,5 & 7,2 & 5,7 & 7,1 \\
50 IFD's & 5,5 & 3,0 & 5,4 & 7,8 & 5,4 & 4,6 & 5,4
\end{tabular}

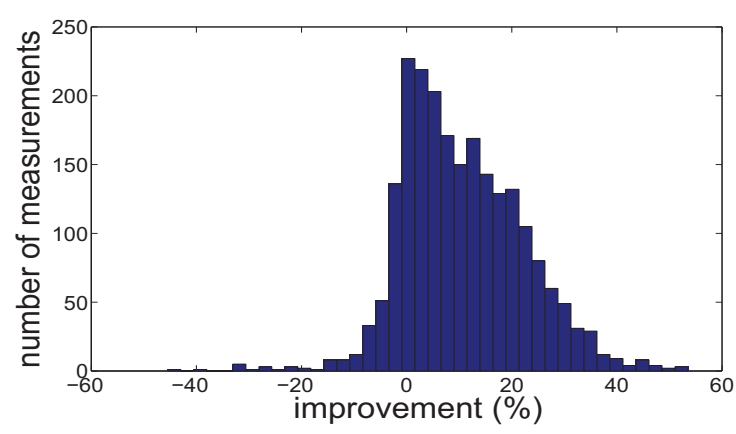

Fig. 3. A histogram of the improvement of the errors of the IFD approximation over the FD approximation, measured with the modified Hausdorff distance.

histogram, there is a small amount of contours which are better approximated using the FD's instead of using the IFD's. This is because the criterion which is used in the algorithm to find an optimal warping function $\theta$ is different from the modified Hausdorff distance.

\section{CONCLUSION}

In this paper shape approximation by first warping the scanning function before calculating the Fourier descriptors was thoroughly tested. It is shown that these shape descriptors, improved Fourier descriptors, are not only theoretically better, but that they also have a significant better performance in practice. Since their good performance, they seem interesting descriptors for a wide range of applications such as silhouette coding. There application to pattern recognition should be further investigated, but seems promising.

\section{ACKNOWLEDGMENT}

The authors would like to thank T.G. Dietterich and the Plant and Botany Department, Oregon State University, for providing the digital herbarium.

\section{REFERENCES}

[1] T. McLellan and J.A. Endler, "The relative success of some methods for measuring and describing the shape of complex objects," Systematic Biology, vol. 47, no. 2, pp. 264-281, 1998.

[2] J.C. Neto, G.E. Meyer, D.D. Jones, and A.K. Samal, "Plant species identification using elliptic fourier leaf shope analysis," Computers and electronics in agriculture, vol. 50, pp. 121134, 2006.

[3] Y. Jeong and R.J. Radke, "Reslicing axially sampled 3d shapes using elliptic fourier descriptors," Medical image Analysis, vol. 11, no. 2, pp. 197-206, April 2007.

[4] M.-A. Charmi, s. Derrode, and F. Ghorbel, "Fourier-based geometric shape prior for snakes," Pattern Recognition Letters, vol. 29, pp. 897-904, 2008.

[5] D. Zhang and G. Lu, "A comparitave study on shape retrieval using fourier descriptors with different shape signatures," Proceedings of International Conference on Intelligent Multimedia and Distance Education, pp. 1-9, 2001.

[6] A. Folkers and H Samet, "Content-based image retrieval using fourier descriptors on a logo database," Proceedings of the 16th International Conference on Pattern Recognition, vol. 3, no. 11, pp. 521-524, August 2002.

[7] D. Zhang and G. Lu., "Review of shape representation and description techniques," Pattern recognition, vol. 37, pp. 119, 2004.

[8] A. Aguedo, M. Nixon, and E. Montiel, "Parameterizing arbitrary shapes via fourier descriptors for evidence-gathering extraction," Computer Vision and Image Understanding, vol. 69, no. 2, pp. 202-221, 1998.

[9] L. Keyes and A. Winstanley, "Fourier descriptors as a genereal classification tool for topographic shapes," Proceedings Irish Machine Vision and Image Processing Conference, pp. 193203, 1999.

[10] M.H. Mahoor and M. Abdel-Mottaleb, "Classification and numbering of teeth indental bitewing images," Pattern Recognition, vol. 38, pp. 577-586, 2005.

[11] W. Philips, "Adaptive warped polynomial contour descriptors," Proceedings of 1995 IEEE International Conference on Systems, Man and Cybernetics, vol. 5, pp. 4638-4643, October 1995. 